\title{
Loops with abelian inner mapping groups: an application of automated deduction $^{\star}$
}

\author{
Michael Kinyon ${ }^{1}$, Robert Veroff ${ }^{2}$, and Petr Vojtěchovskýy ${ }^{\star \star 1}$ \\ ${ }^{1}$ Department of Mathematics, University of Denver, \\ Denver, CO 80208 USA \\ mkinyon@math.du.edu www.math.du.edu/ mkinyon \\ petr@math.du.edu www.math.du.edu/ petr \\ 2 Department of Computer Science, University of New Mexico, \\ Albuquerque, NM 87131 USA \\ veroff@cs.unm.edu WWW.cs.unm.edu/ veroff
}

\begin{abstract}
We describe a large-scale project in applied automated deduction concerned with the following problem of considerable interest in loop theory: If $Q$ is a loop with commuting inner mappings, does it follow that $Q$ modulo its center is a group and $Q$ modulo its nucleus is an abelian group? This problem has been answered affirmatively in several varieties of loops. The solution usually involves sophisticated techniques of automated deduction, and the resulting derivations are very long, often with no higher-level human proofs available.
\end{abstract}

\section{Introduction}

A quasigroup $(Q, \cdot)$ is a set $Q$ with a binary operation $\cdot$ such that for each $x \in Q$, the left translation $L_{x}: Q \rightarrow Q ; y \mapsto y L_{x}=x y$ and the right translation $R_{x}: Q \rightarrow Q ; y \mapsto y R_{x}=y x$ are bijections. A quasigroup is a loop if, in addition, there exists $1 \in Q$ satisfying $1 \cdot x=x \cdot 1=x$ for all $x \in Q$. Standard references for the theory of quasigroups and loops are [1], [3] and [45].

Example 1. The above definition merely says that the multiplication table of a loop is a Latin square (that is, every symbol occurs in every row and in every column precisely once), in which the row labels are duplicated in column 1 and the column labels are duplicated in row 1.

\footnotetext{
* Dedicated to the memory of William McCune (1953-2011).

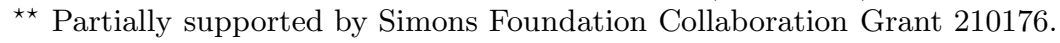


For instance, the following multiplication table defines a loop $Q$ with elements $\{1, \ldots, 6\}$.

\begin{tabular}{l|llllll}
$\cdot$ & 1 & 2 & 3 & 4 & 5 & 6 \\
\hline 1 & 1 & 2 & 3 & 4 & 5 & 6 \\
2 & 2 & 1 & 4 & 3 & 6 & 5 \\
3 & 3 & 4 & 5 & 6 & 1 & 2 \\
4 & 4 & 3 & 6 & 5 & 2 & 1 \\
5 & 5 & 6 & 2 & 1 & 3 & 4 \\
6 & 6 & 5 & 1 & 2 & 4 & 3
\end{tabular}

Note that $Q$ is neither commutative (as $3 \cdot 5=1$ while $5 \cdot 3=2$ ), nor associative (as $3 \cdot(3 \cdot 4)=2$ while $(3 \cdot 3) \cdot 4=1$ ). The left translation $L_{2}$ is the permutation $(1,2)(3,4)(5,6)$, the right translation $R_{3}$ is the permutation $(1,3,5,2,4,6)$.

A quasigroup can be equivalently defined as a set $Q$ with three binary operations - (multiplication), \(left division), and / (right division) satisfying the axioms

$$
x \cdot(x \backslash y)=y=x \backslash(x \cdot y), \quad(x \cdot y) / y=x=(x / y) \cdot y .
$$

Starting with a quasigroup $(Q, \cdot)$, one merely needs to set $x \backslash y=$ $y L_{x}^{-1}$ and $x / y=x R_{y}^{-1}$. Conversely, starting with a three operation quasigroup $(Q, \cdot \backslash, /)$, the above axioms guarantee that all translations $L_{x}, R_{x}$ are bijections of $Q$. For more details on the equivalence of the two definitions, see [9].

Since quasigroups and loops can be equationally defined, they have been fallow ground for the techniques and tools of automated deduction. They appeared already in the milestone paper of Knuth and Bendix [24], and interest in them has continued in the theoremproving community [12 [49]. In more recent years, mathematicians specializing in quasigroups and loops have been making significant use of automated deduction tools $[15,16,17,18,19,20,21,22,23,24,25,26,27,28,29]$ [36, 37, 38,39, 40, 41, 42,43,44]. With the exception of [39] and [40], all of the aforementioned references used Bill McCune's PROver9 [31] or its predecessor OTTER [30].

The purpose of this paper is to report on progress in a large-scale project in the application of automated deduction to loop theory. 
The primary tool has been Prover9, and the search for proofs has relied heavily on the method of proof sketches [48. Proof sketches have been especially effective in this project, in part because of the large number of closely related problems being considered.

In 92 we give the mathematical background and the Main Conjecture and then explain why it is a problem suitable for equational reasoning tools. In 93 we discuss our strategy for attacking the problem with Prover9. As an example, in $₫ 4$ we discuss one particular class of loops for which we were able to solve the problem. Finally in $\$ 5$ we mention other classes of loops for which the solution is known.

\section{The Main Conjecture and the Project}

The left and right translations in a loop $Q$ generate the multiplication group $\operatorname{Mlt}(Q)=\left\langle L_{x}, R_{x} \mid x \in Q\right\rangle$, a subgroup of the group of all bijections on $Q$. The inner mapping group $\operatorname{Inn}(Q)=\{\varphi \in \operatorname{Mlt}(Q) \mid$ $1 \varphi=1\}$ is a subgroup of $\operatorname{Mlt}(Q)$ consisting of all bijections in $\operatorname{Mlt}(Q)$ that fix the element 1 .

A loop $Q$ is said to be an AIM loop (for Abelian Inner Mappings) if $\operatorname{Inn}(Q)$ is an abelian group. AIM loops are the main subject of this investigation.

A nonempty subset $S$ of a loop $Q$ is a subloop $(S \leq Q)$ if it is closed under the three operations $\cdot, \backslash, /$. A subloop $S$ of $Q$ is normal $(S \unlhd Q)$ if $S \varphi=\{s \varphi \mid s \in S\}$ is equal to $S$ for every $\varphi \in \operatorname{Inn}(Q)$.

To save space, we will often denote $x \cdot y$ by $x y$, and we will use . to indicate the priority of multiplication. For instance, $x \cdot y z$ stands for $x \cdot(y \cdot z)$.

The left, right, and middle nucleus of a loop $Q$ are defined, respectively, by

$$
\begin{array}{ll}
N_{\lambda}(Q)=\{a \in Q \mid a x \cdot y=a \cdot x y, & \forall x, y \in Q\}, \\
N_{\rho}(Q)=\{a \in Q \mid x y \cdot a=x \cdot y a, & \forall x, y \in Q\}, \\
N_{\mu}(Q)=\{a \in Q \mid x a \cdot y=x \cdot a y, & \forall x, y \in Q\},
\end{array}
$$

and the nucleus is $N(Q)=N_{\lambda}(Q) \cap N_{\rho}(Q) \cap N_{\mu}(Q)$. It is not hard to show that each of these nuclei is a subloop.

The commutant of a loop $Q$ is the set

$$
C(Q)=\{a \in Q \mid a x=x a \quad \forall x \in Q\},
$$


which is not necessarily a subloop. The center of $Q$ is

$$
Z(Q)=C(Q) \cap N(Q) .
$$

The center is always a normal subloop.

Thus the nucleus $N(Q)$ consists of all elements $a \in Q$ that associate with all $x, y \in Q$, the commutant $C(Q)$ consists of all elements $a \in Q$ that commute with all $x \in Q$, and the center $Z(Q)$ consists of all elements $a \in Q$ that commute and associate with all $x, y \in Q$.

For a general loop $Q$, the inclusions $N_{\lambda}(Q) \leq N(Q), N_{\rho}(Q) \leq$ $N(Q), N_{\mu}(Q) \leq N(Q), N(Q) \leq Z(Q)$ and $C(Q) \leq Z(Q)$ hold, but not necessarily the equalities.

Given a normal subloop $S$ of a loop $Q$, denote by $Q / S$ the factor loop $Q$ modulo $S$ whose elements are the subsets (left cosets) $x S=$ $\{x s \mid s \in S\}$ for $x \in Q$, and where we multiply according to $x S \cdot y S=$ $(x \cdot y) S$.

Now define $Z_{0}(Q)=\{1\}$, and for $i \geq 0$ let $Z_{i+1}(Q)$ be the preimage of $Z\left(Q / Z_{i}(Q)\right)$ under the canonical projection $\pi_{i}: Q \rightarrow$ $Q / Z_{i}(Q) ; x \mapsto x Z_{i}(Q)$. Note that $\{1\}=Z_{0}(Q) \leq Z_{1}(Q) \leq Z_{2}(Q) \leq$ $\cdots \leq Q$. The loop $Q$ is (centrally) nilpotent of class $n$, written $\mathrm{c} \ell(Q)=n$, if $Z_{n-1}(Q) \neq Q$ and $Z_{n}(Q)=Q$.

Example 2. Let $Q$ be the loop from Example 1. A short computer calculation shows that the multiplication group $\operatorname{Mlt}(Q)$ has size 24, and the inner mapping group $\operatorname{Inn}(Q)$ consists of the permutations ()$,(3,4),(5,6),(3,4)(5,6)$. In particular, $\operatorname{Inn}(Q)$ is a group of size 4 , hence an abelian group, and $Q$ is therefore an AIM loop.

$Q$ has only three subloops, namely the subsets $\{1\},\{1,2\}$ and $\{1,2,3,4,5,6\}$. It so happens that all four nuclei, the commutant and the center of $Q$ are equal to the normal subloop $\{1,2\}$. We have $Z_{0}(Q)=1, Z_{1}(Q)=Z(Q)=\{1,2\}$ and $Z_{2}(Q)=\{1,2,3,4,5,6\}$ (because $Q / Z_{1}(Q)$ is an abelian group). Thus $\mathrm{c} \ell(Q)=2$.

Recall (or see [46, Thm. 7.1]) that if $Q$ is a group then

$$
\operatorname{Inn}(Q) \text { is isomorphic to } Q / Z(Q) \text {. }
$$

This result cannot be generalized to loops. For instance, we saw in Example 2 that there is a loop $Q$ of size 6 with $\operatorname{Inn}(Q)$ of size 4 and $Q / Z(Q)$ of size $6 / 2=3$. 
Now, if $Q$ is a group, we deduce from (11) that

$$
\mathrm{c} \ell(\operatorname{Inn}(Q)) \leq n \text { if and only if } \mathrm{c} \ell(Q) \leq n+1 .
$$

Neither of the two implications in (2) generalizes to loops. Indeed, Vesanen found a loop $Q$ of size 18 with $\mathrm{c} \ell(Q)=3 \operatorname{such}$ that $\operatorname{Inn}(Q)$ is not even nilpotent, much less of nilpotency class at most 2; see [14]. To falsify the other implication, we will see below that there exist loops with $\mathrm{c} \ell(\operatorname{Inn}(Q))=n$ but with $\mathrm{c} \ell(Q) \neq n+1$. (Note, however, that Niemenmaa was able to prove recently that if $Q$ is finite and $\operatorname{Inn}(Q)$ is nilpotent then $Q$ is at least nilpotent [34.)

Using $n=1$ in (2), we see that for a group $Q$

$\operatorname{Inn}(Q)$ is abelian (that is, $Q$ is an AIM loop) if and only if $\mathrm{c} \ell(Q) \leq 2$.

Does this statement generalize to loops? The answer is of importance in loop theory because it sheds light on loops of nilpotency class 2 , in some sense the most immediate generalization of abelian groups to loops.

Bruck showed in [2] that one implication of (3) holds for all loops: if $Q$ is a loop with $\mathrm{c} \ell(Q) \leq 2$ then $Q$ is an AIM loop. For a long time, it was conjectured that the other implication of (13) also holds, and much work in loop theory was devoted to this problem [6] [13] [35]. However, in 2004, Csörgö [4] disproved (3) by constructing an AIM loop $Q$ (of size 128) with $\mathrm{c} \ell(Q)=3$.

Consequently, AIM loops $Q$ with $\mathrm{c} \ell(Q) \geq 3$ have come to be called loops of Csörgö type. Additional constructions of loops of Csörgö type followed in rapid succession [7] [8] [33]. We remark that it is still not known if there exists an AIM loop $Q$ with $\mathrm{c} \ell(Q)>3$.

What can be salvaged from the statement (3) for loops? Based on the structure of all known loops of Csörgő type, the first-named author has been offering the following structural conjecture in various talks and presentations. This is the first published statement of the conjecture.

Main Conjecture. Let $Q$ be an AIM loop. Then $Q / N(Q)$ is an abelian group and $Q / Z(Q)$ is a group. In particular, $Q$ is nilpotent of class at most 3 . 
Three remarks are worth making here. First, the primary assertion of the Main Conjecture is actually somewhat stronger than the "in particular" part, that is, having nilpotency class 3 does not necessarily imply $Q / N(Q)$ is abelian or $Q / Z(Q)$ is a group. Second, note that the Main Conjecture makes no reference to cardinality of the loop $Q$. It is certainly conceivable that the conjecture holds for all finite loops but that there is some infinite counterexample. Finally, it is tacit in the statement of the conjecture that the nucleus $N(Q)$ is a normal subloop of $Q$ (else the factor loop $Q / N(Q)$ cannot be formed). This is not true for arbitrary loops but is easy to show for AIM loops.

From the discussion so far, it may seem that the Main Conjecture is too high order to be attacked fruitfully by the methods of automated deduction. However, the hypotheses and conclusions of the conjecture can be given purely equationally as we now describe.

Bruck showed [3] that the inner mapping group is generated by three kinds of mappings that measure deviations from associativity and commutativity, namely,

$$
\operatorname{Inn}(Q)=\left\langle R_{x, y}, T_{x}, L_{x, y} ; x, y \in Q\right\rangle,
$$

where

$$
R_{x, y}=R_{x} R_{y} R_{x y}^{-1}, \quad T_{x}=R_{x} L_{x}^{-1}, \quad L_{x, y}=L_{x} L_{y} L_{y x}^{-1} .
$$

Since a group is abelian if and only if any two of its generators commute, we immediately obtain the following characterization of AIM loops:

Lemma 1. A loop $Q$ is an AIM loop if and only if the following identities hold:

$$
\begin{aligned}
T_{x} T_{y} & =T_{x} T_{y}, \\
L_{x, y} L_{z, w} & =L_{z, w} L_{x, y}, \\
R_{x, y} R_{z, w} & =R_{z, w} R_{x, y}, \\
L_{x, y} T_{z} & =T_{z} L_{x, y}, \\
R_{x, y} T_{z} & =T_{z} R_{x, y}, \\
L_{x, y} R_{z, w} & =R_{z, w} L_{x, y}
\end{aligned}
$$

for all $x, y, z, w \in Q$. 
To encode the conclusions of the Main Conjecture, it is useful to introduce two more functions, the associator

$$
[x, y, z]=(x \cdot y z) \backslash(x y \cdot z)
$$

and the commutator

$$
[x, y]=(y x) \backslash(x y)
$$

that is, $(x \cdot y z)[x, y, z]=x y \cdot z$ and $y x \cdot[x, y]=x y$. The former is a measure of nonassociativity and the latter is a measure of noncommutativity. Different conventions are possible for each of these functions, e.g. one could use $(x y \cdot z) /(x \cdot y z)$ as a definition of associator. Our convention is the traditional one [3]. Note that an element $a \in Q$ is in the left nucleus $N_{\lambda}(Q)$ if and only if $[a, x, y]=1$ for all $x, y \in Q$, and that the other nuclei are similarly characterized.

The following is now a straightforward consequence of the definitions.

Lemma 2. Let $Q$ be a loop. Then

(i) If $N(Q) \unlhd Q$ then $Q / N(Q)$ is an abelian group if and only if the following identities hold:

$$
\begin{aligned}
{[[x, y, z], u, v] } & =[u,[x, y, z], v]=[u, v,[x, y, z]]=1 \\
{[[x, y], z, u] } & =[z,[x, y], u]=[z, u,[x, y]]=1
\end{aligned}
$$

for all $x, y, z, u, v \in Q$;

(ii) $Q / Z(Q)$ is a group if and only if the following identities hold:

$$
\begin{aligned}
{[[x, y, z], u, v]=[u,[x, y, z], v] } & =[u, v,[x, y, z]]=1 \\
{[[x, y, z], u] } & =1
\end{aligned}
$$

for all $x, y, z, u, v \in Q$.

Proof. Let $S \unlhd Q$. The following conditions are equivalent: $(x S \cdot y S)$. $z S=x S \cdot(y S \cdot z S),(x y \cdot z) S=(x \cdot y z) S,((x \cdot y z) \backslash(x y \cdot z)) S=S$, $[x, y, z] S=S,[x, y, z] \in S$. Thus $Q / S$ is a group if and only if $[x, y, z] \in S$ for every $x, y, z \in Q$. Similarly, $Q / S$ is commutative if and only if $[x, y] \in S$ for every $x, y \in Q$. 
The condition $[[x, y, z], u, v]=[u,[x, y, z], v]=[u, v,[x, y, z]]=1$ says that $[x, y, z] \in N(Q)$ for all $x, y, z \in Q$, and the condition $[[x, y], z, u]=[z,[x, y], u]=[z, u,[x, y]]=1$ says that $[x, y] \in N(Q)$ for all $x, y \in Q$. This proves (i).

Concerning (ii), the condition $[[x, y, z], u]=1$ says that $[x, y, z] \in$ $C(Q)$ for every $x, y, z \in Q$. Since $Z(Q)=N(Q) \cap C(Q)$, we are done.

In Figure 1 we give a basic Prover9 input file for the Main Conjecture. To encode loops, we use the definition with three binary operations ·, \and /. The clauses labeled "obvious compatibility" are not necessary, but are included to help the search. Note how the assumptions on AIM loops correspond to the identities of Lemma 1, and how the goals correspond to the identities of Lemma 2 .

A resolution one way or the other of the full Main Conjecture would be a major milestone in loop theory, alas, the answer remains elusive. Nevertheless we managed to confirm the Main Conjecture for several classes of loops. We describe one of the successful cases in $\$ 4$ and list others in $\$ 5$.

\section{$3 \quad$ The Strategy}

Our search for proofs for the various AIM cases involves sequences of PROVER9 experiments that rely heavily on the use of hints [47] and on the method of proof sketches [48]. Under the hints strategy, a generated clause is given special consideration if it matches (subsumes) a user-supplied hint clause. In Prover9, the actions associated with hint matching are controllable by the user, but the most typical action is to give hint matchers the highest priority in the proof search.

A proof sketch for a theorem $T$ is a sequence of clauses giving a set of conditions sufficient to prove $T$. In the ideal case, a proof sketch consists of a sequence of lemmas, where each lemma is fairly easy to prove. In any case, the clauses of a proof sketch identify potentially notable milestones on the way to finding a proof. From a strategic standpoint, it is desirable to recognize when we have achieved such milestones and to adapt the continued search for a proof accordingly. In particular, we want to focus our attention on 


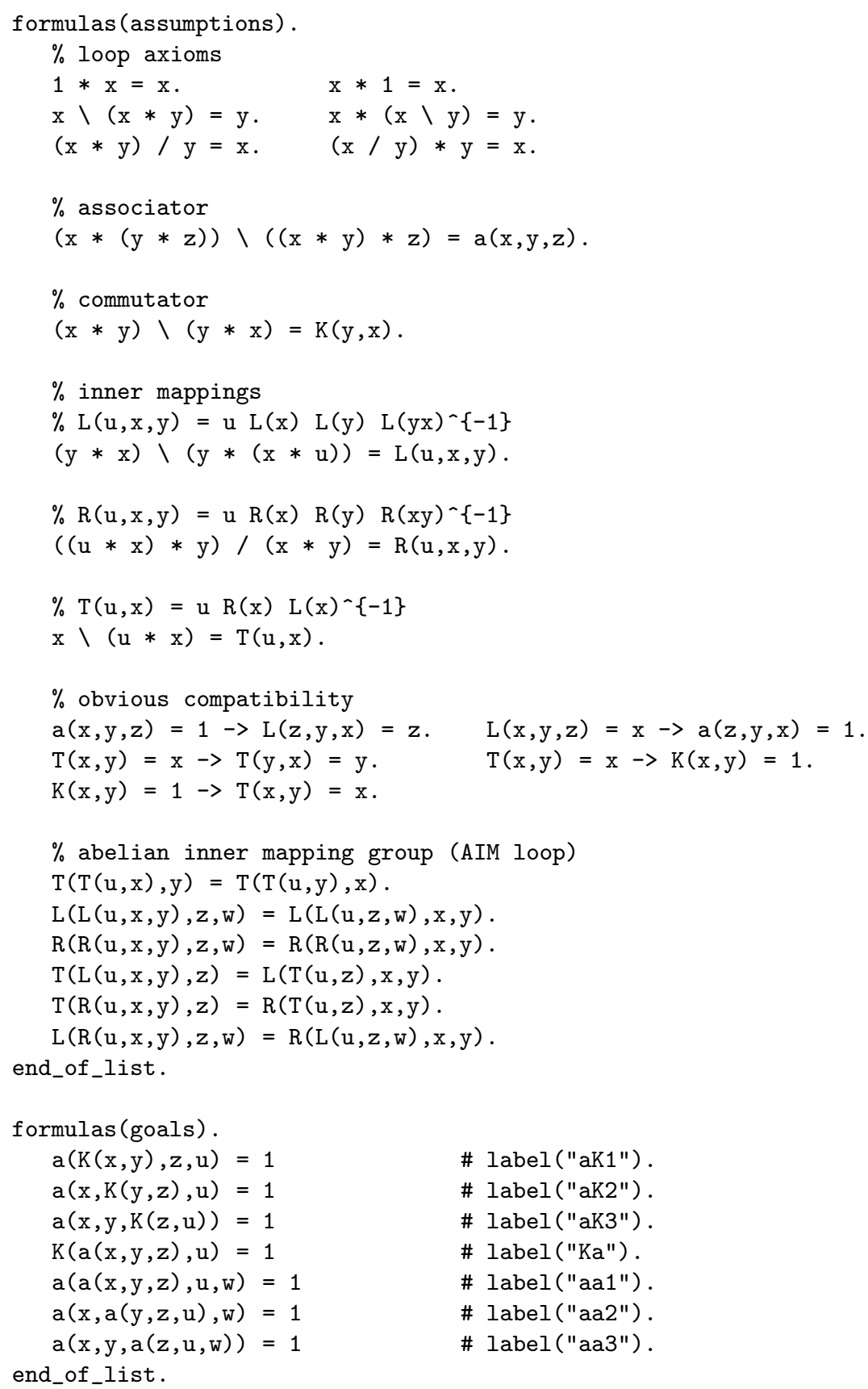

Fig. 1. A Prover9 input file for the problem "In an AIM loop $Q$, is $Q / N(Q)$ an abelian group and is $Q / Z(Q)$ a group?" 
such milestone results and pursue their consequences sooner rather than later. The hints mechanism provides a natural and effective way to take full advantage of proof sketches in the search for a proof.

The use of hints is additive in the sense that hints from multiple proof sketches or from sketches for different parts of a proof can all be included at the same time. For this reason, hints are particularly valuable for "gluing" subproofs together and completing partial proofs, even when wildly different search strategies were used to find the individual subproofs.

In [48, we consider how the generation and use of proof sketches, together with the sophisticated strategies and procedures supported by an automated reasoning program such as PROVER9, can be used to find proofs to challenging theorems, including open questions. The general approach is to find proofs with additional assumptions and then to systematically eliminate these assumptions from the input set, using all previous proofs as hints. It also can be very effective to include as proof sketches proofs of related theorems in the same area of study. In the AIM study, for example, proofs for the LC case (see Section (4) were found by first proving the LC case with the additional assumption "left inner maps preserve inverses",

$$
\mathrm{L}(\mathrm{x}, \mathrm{y}, \mathrm{z}) \backslash 1=\mathrm{L}(\mathrm{x} \backslash 1, \mathrm{y}, \mathrm{z}) .
$$

The resulting proofs, together with proofs of other previously proved cases, were included as proof sketches in the search that found the proofs for LC alone.

Our strategy for searching for AIM proofs is based on the following two observations.

- Proofs for the various special cases tend to share several key steps. This makes these problems especially amenable to the use of previously found proofs as hints.

- Proof searches in this problem area tend to be especially sensitive to the underlying lexical ordering of terms. This is due primarily to the resulting effect on the demodulation (rewriting) of deduced clauses.

Addressing the second observation, we can run multiple searches, trying each of several term orderings in turn. But rather than simply running each of these searches as independent attempts, we can 
leverage Prover9's hints mechanism to take advantage of any apparent progress made in previous searches. In particular, after running a search with one term ordering, we can gather the derived clauses that match hints and include these as input assumptions for the following runs.

The second-named author has automated this approach with a utility called p9loop. P9loop takes as input an ordinary PROVER9 input file (including hints) and a list of term ordering directives and proceeds as follows.

- Run Prover9 with a term ordering from the list until either a proof is found or a user-specified processing limit is reached.

- If no proof has been found, restart PROvER9 with the next term ordering from the list, including all of the previous hint matchers as additional input assumptions.

We have had numerous successes using this approach, sometimes finding proofs that rely on several p9loop iterations. We have, for example, found proofs after iterating through over 50 term orderings, with as many as 30 of the iterations contributing to the found proof.

We note that the final iteration of a successful p9loop execution generally results in a proof that includes assumptions derived in previous Prover9 runs and that we do not immediately have the derivations of these assumptions. Furthermore, this property may be nested in that an assumption from a previous run may in turn rely on assumptions from even earlier runs. In order to get a complete

proof of the final theorem, we use PROVER9 to recover the missing derivations by systematically eliminating these assumptions in a way that is analogous to the general proof sketches method.

\section{LC Loops}

A loop $Q$ is said to be an $L C$ loop if it satisfies any of the following equivalent identities:

$$
\begin{aligned}
& x(x(y z))=(x(x y)) z \\
& x(x(y z))=((x x) y) z, \\
& (x x)(y z)=(x(x y)) z, \\
& x(y(y z))=(x(y y)) z
\end{aligned}
$$


for all $x, y, z \in Q$. LC loops were introduced by Fenyves [10] as one of the loops of Bol-Moufang type. They were studied in more detail in [41] where the equivalence of the identities above was proven.

We have been able to establish the Main Conjecture in the special case of LC loops, that is, we have the following theorem.

Theorem 1. Let $Q$ be an AIM LC loop. Then $Q / N(Q)$ is an abelian group and $Q / Z(Q)$ is a group. In particular, $Q$ is nilpotent of class at most 3 .

It sometimes is desirable to obtain a humanized proof of a result first found by means of automated deduction tools. Generally speaking, the process of "humanization" can give some higher-level insights into the structure being studied and can often result in a simpler (from a human perspective) proof than the one originally generated by an automated deduction tool. Many of the references in the bibliography feature humanized proofs.

There are various varieties of loops with abelian inner mappings for which the proof of the conjecture will be worth humanizing. We list a few of these in the next section. However, that desire for a human proof is tempered by the law of diminishing returns. In some cases, a human proof may not be worth the time and effort put into translating the automated proof. For LC loops, we run right into this issue.

We often prefer to produce automated proofs that are strictlyforward derivations of the theorems and that are free of any applications of demodulation. This sometimes is for purposes of presentation, but most often it's because we find that these proofs make better proof sketches for future searches, especially in a larger study such as the AIM project. In PROVER9, these constraints can be satisfied with the directives set (restrict_denials) and clear(back_demod) respectively. If the input list formulas (demodulators) is empty, including the directive clear(back_demod) ensures that no clauseinput or derived - will be used as a demodulator.

Here is the data for the strictly-forward, demodulation-free proofs of the seven LC goals: 


\begin{tabular}{ccc}
\multicolumn{3}{c}{ Goal Length Level } \\
\hline Ka & 2192 & 222 \\
aK1 & 2191 & 221 \\
aK2 & 2199 & 224 \\
aK3 & 2394 & 276 \\
aa1 & 2842 & 321 \\
aa2 & 2842 & 321 \\
aa3 & 2841 & 320
\end{tabular}

Of course, the seven proofs have many clauses in common and some proofs are virtually identical. For example, it is known that in LC loops, the left and middle nuclei coincide. The goals aa1 and aa2 state that associators are in those nuclei, so it is not surprising that their proof lengths and levels are the same. The proofs (and corresponding input file) can be found on this paper's associated Web page 23].

Since at present, the authors think that the classes of loops discussed in the next section will be more worthy of human translation, any attempt to do so for LC loops has been postponed.

\section{$5 \quad$ Further Remarks}

Within certain varieties of loops, it is known that there are no loops of Csörgö type. In other words, given an AIM loop $Q$ in that variety, $Q$ is necessarily nilpotent of class at most 2 .

In order to formulate the problem of showing that an AIM loop in a particular variety is centrally nilpotent of class at most 2 , it is only necessary to add the defining equations of the variety to the assumptions and to add one more goal:

$$
[[x, y], z]=1
$$

for all $x, y, z$. Indeed, we already know from the identities of Lemma 2 that $Q / Z(Q)$ is a group, that $[x, y] \in N(Q)$, and the last goal says that $[x, y] \in C(Q)$, so $[x, y] \in Z(Q)=C(Q) \cap N(Q)$ and $Q / Z(Q)$ is an abelian group, i.e., $\mathrm{c} \ell(Q) \leq 2$.

Figure 2 summarizes what is known about the Main Conjecture and about the above-mentioned problem in several well-studied varieties of loops. The varieties include abelian groups, groups, Steiner 


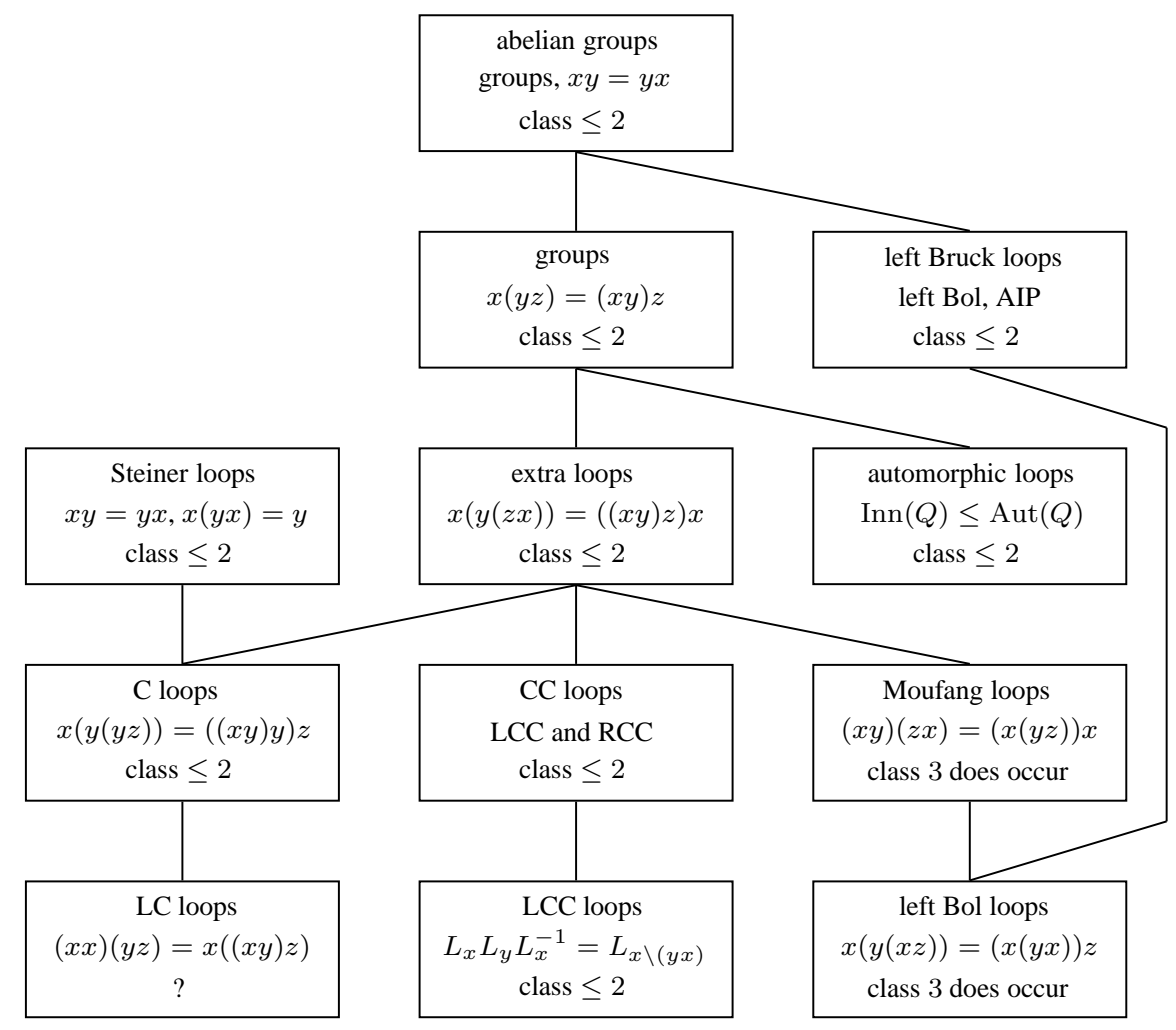

Fig. 2. Well-studied varieties of loops in which the Main Conjecture is true. 
loops (defined by the identities $x y=y x, x(y x)=y$ ), extra loops (defined by $x(y(z x))=((x y) z) x)$, automorphic loops (loops where all inner mappings are automorphisms), $C$ loops (defined by $((x y) y) z=$ $x(y(y z))$ ), conjugacy closed (or $C C$ ) loops (loops in which $L_{x} L_{y} L_{x}^{-1}$ is a left translation and $R_{x} R_{y} R_{x}^{-1}$ is a right translation for every $x$, $y)$, LC loops, left Bol loops (defined by $x(y(x z))=(x(y x)) z)$, left conjugacy closed (or $L C C$ ) loops (loops in which $L_{x} L_{y} L_{x}^{-1}$ is a left translation for every $x, y$ ), and left Bruck loops (that is, left Bol loops with the automorphic inverse property (or AIP) $(x y)^{-1}=x^{-1} y^{-1}$ ). To keep the figure legible, we omitted all dual varieties (RC loops, right Bol loops, RCC loops, right Bruck loops) for which analogous results hold.

The varieties in Figure 2 are listed with respect to inclusion, with smaller varieties higher up. For instance, every Moufang loop is a left Bol loop.

All varieties in Figure 2 satisfy the Main Conjecture. Moreover, some varieties have the stronger property discussed above that an AIM loop $Q$ satisfies $\mathrm{c} \ell(Q) \leq 2$. This is indicated by "class $\leq 2$ " in the figure. The already known cases where $\mathrm{c} \ell(Q) \leq 2$ are AIM LCC loops [5] and AIM left Bruck loops [40].

All other cases indicated in the figure - automorphic loops, Moufang loops, left Bol loops and C loops-comprise new results which are part of this project. Their proofs will appear elsewhere, often in humanized form. Another new result is that an AIM Moufang loop $Q$ satisfies $\mathrm{c} \ell(Q) \leq 2$ if $Q$ is uniquely 2-divisible, that is, the mapping $x \mapsto x^{2}$ is a bijection of $Q$. (Previously it was known that an AIM Moufang loop that is uniquely 2-divisible and finite satisfies $c \ell(Q) \leq 2$, and that there is an AIM Moufang loop $Q$ of size $2^{14}$ satisfying $\mathrm{c} \ell(Q)=3$ [33.) The same result holds for uniquely 2-divisible AIM left Bol loops.

Despite some serious effort, we have not been able to decide if an AIM LC loop $Q$ satisfies $c \ell(Q) \leq 2$ (indicated in the figure by "?"); we believe that it does.

Finally, all other claims contained within the figure are consequences of the inclusions. 


\section{References}

1. Belousov, V. D.: Foundations of the Theory of Quasigroups and Loops. (in Russian) Izdat. Nauka, Moscow (1967).

2. Bruck, R. H.: Contributions to the theory of loops. Trans. Amer. Math. Soc. 60, 245-354 (1946).

3. Bruck, R. H.: A Survey of Binary Systems. Springer-Verlag, Berlin-GöttingenHeidelberg (1971).

4. Csörgö, P.: Abelian inner mappings and nilpotency class greater than two. European J. Combin. 28, 858-867 (2007).

5. Csörgö, P. and Drápal, A.: Left conjugacy closed loops of nilpotency class two. Results Math. 47, 242-265 (2005).

6. Csörgö, P. and Kepka, T.: On loops whose inner permutations commute. Comment. Math. Univ. Carolin. 45, 213-221 (2004).

7. Drápal, A. and Kinyon, M. K.: Buchsteiner loops: associators and constructions. arXiv/0812.0412.

8. Drápal, A. and Vojtěchovský, P.: Explicit constructions of loops with commuting inner mappings. European J. Combin. 29, 1662-1681 (2008).

9. Evans, T.: Homomorphisms of non-associative systems. J. London Math. Soc. 24, 254-260 (1949).

10. Fenyves, F.: Extra loops II. On loops with identities of Bol-Moufang type. Publ. Math. Debrecen, 16, 187-192 (1969).

11. The GAP Group: GAP - Groups, Algorithms, and Programming, Version 4.4.10; 2007. Www.gap-system.org

12. Hullot, J.-M.: A catalogue of canonical term rewriting systems. Technical Report CSC 113, SRI International (1980).

13. Kepka, T.: On the abelian inner permutation groups of loops. Comm. Algebra 26, 857-861 (1998).

14. Kepka T. and Phillips, J. D.: Connected transversals to subnormal subgroups. Comment. Math. Univ. Carolin. 38, 223-230 (1997).

15. Kepka, T., Kinyon, M. K. and Phillips, J. D.: The structure of F-quasigroups. J. Algebra 317, 435-461 (2007).

16. Kinyon, M. K., Kunen, K. and Phillips, J. D.: Every diassociative A-loop is Moufang. Proc. Amer. Math. Soc. 130, 619-624 (2002).

17. Kinyon, M. K., Kunen, K., and Phillips, J. D.: A generalization of Moufang and Steiner loops. Algebra Universalis 48, 81-101 (2002).

18. Kinyon, M. K., Kunen, K. and Phillips, J. D.: Diassociativity in conjugacy closed loops. Comm. Algebra 32, 767-786 (2004).

19. Kinyon, M. K. and Kunen, K.: The structure of extra loops. Quasigroups and Related Systems 12, 39-60 (2004).

20. Kinyon, M. K. and Kunen, K.: Power-associative, conjugacy closed loops. J. Algebra 304, 679-711 (2006).

21. Kinyon, M. K., Phillips, J. D. and Vojtěchovský, P.: C-loops: extensions and constructions. J. Algebra Appl. 6, 1-20 (2007).

22. Kinyon, M. K., Phillips, J. D. and Vojtěchovský, P.: When is the commutant of a Bol loop a subloop? Trans. Amer. Math. Soc. 360, 2393-2408 (2008).

23. Kinyon, M. K., Veroff, R. and Vojtěchovský, P.: Loops with abelian inner mapping groups: an application of automated deduction (Web support). (2012) Www.cs.unm.edu/ veroff/AIM_LC/ 
24. Knuth, D. E. and Bendix, P. B.: Simple word problems in universal algebras. In J. Leech (ed.), Proceedings of the Conference on Computational Problems in Abstract Algebras, Oxford, 1967, Pergamon Press, Oxford, 263-298 (1970).

25. Kunen, K.: Moufang quasigroups. J. Algebra 183, 231-234 (1996).

26. Kunen, K.: Quasigroups, loops, and associative laws. J. Algebra 185, 194-204 (1996).

27. Kunen, K.: Alternative loop rings. Comm. Algebra 26, 557-564 (1998).

28. Kunen, K.: G-Loops and permutation groups. J. Algebra 220, 694-708 (1999).

29. Kunen, K.: The structure of conjugacy closed loops. Trans. Amer. Math. Soc. 352, 2889-2911 (2000).

30. McCune, W.: Otter 3.0 Reference Manual and Guide. Tech. Report ANL-94/6, Argonne National Laboratory, Argonne, IL (1994). See also wWw.mcs.anl.gov/AR/otter/

31. McCune, W.: Prover9, version 2009-02A, www.cs.unm.edu/ mccune/prover9/

32. Nagy, G. and Vojtěchovský, P.: LOOPS: Computing with quasigroups and loops in GAP - a GAP package, version 2.0.0, (2008). www.math.du.edu/loops

33. Nagy, G. P. and Vojtěchovský, P.: Moufang loops with commuting inner mappings. J. Pure Appl. Algebra 213, 2177-2186 (2009).

34. Niemenmaa, M.: Finite loops with nilpotent inner mapping groups are centrally nilpotent. Bull. Aust. Math. Soc. 79, 109-114 (2009).

35. Niemenmaa, M. and Kepka, T.: On connected transversals to abelian subgroups in finite groups. Bull. London Math. Soc. 24, 343-346 (1992).

36. Phillips, J. D.: A short basis for the variety of WIP PACC-loops. Quasigroups Related Systems 14, 73-80 (2006).

37. Phillips, J. D.: The Moufang laws, global and local. J. Algebra Appl. 8, 477-492 (2009).

38. Phillips, J. D. and Shcherbacov, V. A.: Cheban loops. J. Gen. Lie Theory Appl. 4 (2010), Art. ID G100501, 5 pp.

39. Phillips, J. D. and Stanovský, D.: Automated theorem proving in quasigroup and loop theory. AI Commun. 23, 267-283 (2010).

40. Phillips, J. D. and Stanovský, D.: Bruck loops with abelian inner mapping groups. Comm. Alg., to appear.

41. Phillips, J. D. and Vojtěchovský, P.: The varieties of loops of Bol-Moufang type. Algebra Universalis 54, 259-271 (2005).

42. Phillips, J. D. and Vojtěchovský, P.: The varieties of quasigroups of Bol-Moufang type: an equational reasoning approach. J. Algebra 293, 17-33 (2005).

43. Phillips, J. D. and Vojtěchovský, P.: C-loops: an introduction. Publ. Math. Debrecen 68, 115-137 (2006).

44. Phillips, J. D. and Vojtěchovský, P.: A scoop from groups: equational foundations for loops. Comment. Math. Univ. Carolin. 49, 279-290 (2008).

45. Pflugfelder, H. O.: Quasigroups and Loops: Introduction. Sigma Series in Pure Math. 8, Heldermann Verlag, Berlin (1990).

46. Rotman, J. J.: An Introduction to the Theory of Groups. 4th edition. SpringerVerlag, New York (1995).

47. Veroff, R.: Using hints to increase the effectiveness of an automated reasoning program: case studies. J. Automated Reasoning 16, 223-239 (1996).

48. Veroff, R.: Solving open questions and other challenge problems using proof sketches. J. Automated Reasoning 27, 157-174 (2001).

49. Zhang, H., Bonacina, M. P. and Hsiang, J.: PSATO: a distributed propositional prover and its application to quasigroup problems. J. Symbolic Computation 21, 543-560 (1996). 